Original Paper

\title{
The Myth of Blunted Gamers: No Evidence for Desensitization in Empathy for Pain after a Violent Video Game Intervention in a Longitudinal fMRI Study on Non-Gamers
}

\author{
Simone Kühn ${ }^{a, b}$ Dimitrij Kugler ${ }^{b}$ Katharina Schmalen ${ }^{a} \quad$ Markus Weichenberger ${ }^{a}$ \\ Charlotte Witt $^{\mathrm{a}}$ Jürgen Gallinat ${ }^{b}$
}

aMax Planck Institute for Human Development, Center for Lifespan Psychology, Berlin, bUniversity Clinic Hamburg-Eppendorf, Clinic and Policlinic for Psychiatry and Psychotherapy, Hamburg, Germany

\section{Key Words}

Violent video games $•$ Aggression $•$ Empathy $• \mathrm{fMRI} \cdot$ Training study

\begin{abstract}
Background/Aims: It is a common concern in the research field and the community that habitual violent video gaming reduces empathy for pain in its players. However, previous fMRI studies have only compared habitual game players against control participants crosssectionally. However the observed pattern of results may be due to a priori differences in people who become gamers and who not. In order to derive the causal conclusion that violent video game play causes desensitisation, longitudinal studies are needed. Methods: Therefore we conducted a longitudinal fMRI intervention study over 16 weeks. Participants were randomly assigned to 1) play a violent video game (Grand Theft Auto 5), 2) perform a social life simulation game (The Sims 3) 30 min/day for 8 weeks, 3) serve as passive control. To assess empathy processing, participants were exposed to painful and non-painful stimuli (e.g. someone cutting a cucumber with or without hurting herself) either as real photographs or video-game like depictions in a 3T MRI scanner before and after the training intervention as well as two months after training. Results: We did not find any evidence for desensitization in the empathy network for pain in the violent video game group at any time point. Conclusions: The present results provide strong evidence against the frequently proclaimed negative effects of playing violent video games and will therefore help to communicate a more realistic scientific perspective of the effects of violent video gaming in real life.
\end{abstract}

\section{Introduction}

The public concern that violent video games may promote aggressive behaviour or reduce empathy in its players is pervasive and given the popularity of these games, their psychological impact is an important question for society at large. Unfortunately the research 
field seems to be divided into two camps, one postulates a direct causal role of violent video gaming in aggression [1, 2]. In the light of this so-called General Aggression Model (GAM) desensitization against violent content and a decrease of empathy and prosocial behaviour has been posited as a result of playing violent video games $[3,4]$. The other camp criticizes this theory and argues based on discrepancies between its resulting predictions and actual statistics on violence $[5,6]$. They suggest that the conclusions of the GAM are the result of selective reporting and publication biases [7].

Another major critique is the study design that has commonly been used to test the GAM. Many of the studies exploring changes in aggression or prosocial behaviour due to video gaming expose participants to only short durations of video game play ranging from four minutes to maximally two hours (mean=22 min, median=15 min, when considering all experimental studies reviewed in two of the recent major meta-analyses in the field [8, 9]). In addition to this, the crucial research question in our view is not whether people are more aggressive or less empathic after a few minutes to hours of game play, but rather what the long-term effects of video game playing are. Unfortunately, such longitudinal studies, in which participants are exposed over extensive periods of time, are rare. However, these studies are urgently needed in particular if one intends to inform the general public about real-life consequences of violent video game play.

Within the scope of the present study we wanted to fill this gap and aimed at investigating whether there are effects of long-term violent video gaming on brain activation that would resemble the pattern predicted for desensitization. Previous behavioural studies that trained only for minutes to hours reveal an inconsistent picture, with some demonstrating desensitization of heart rate, galvanic skin response $[10,11]$ or event related potentials (P300) [12] in response to violent stimuli and others not demonstrating these desensitization effects albeit using very similar outcome measures and stimuli $[13,14]$.

To our knowledge there are no studies that investigated the effects of violent video games on desensitisation of brain responses that exposed participants to considerable training durations. Instead several studies investigated desensitization effects in habitual gamers compared to no-gamers, showing amplitude reductions in event-related potentials (P300) in response to violent pictures [15]. In the first fMRI study small differences were shown when comparing habitual violent video gamers and non-gamers who were confronted with images from the IAPS picture set and screenshots from a violent video game [16]. However, the effects observed on the game-specific stimuli are difficult to interpret since the familiarity of the stimuli differed between groups since gamers were more familiar with the game-related picture content. Two later fMRI studies with a similar design but using stimuli that were not related to video game play (IAPS, line drawings) did not show any evidence for desensitization in habitual violent video gamers $[17,18]$. Similarly, a recent study using photographs of painful and non painful situations did not reveal any differences in empathy for pain [19]. Another fMRI study using virtual violent films and real violent films and compared violent video gamers against controls likewise did not find evidence for desensitization nor did they find specific differences in processing between virtual and reallife violence in gamers [20].

A major problem of these prior study designs comparing habitual violent video gamers against control participants cross-sectionally is that they do not allow any causal conclusions. It is unclear whether potential differences in processing of violent stimuli are caused by the habitual video gaming itself or whether these differences in processing of violent stimuli predisposed the participants to start and/ or maintain the violent game play habit. Conceivably participants who are not very sensitive to violent stimuli and seeing somebody else in pain may enjoy violent video games more. The only study design that can unravel the directionality of the effects and demonstrate that violent video game playing actually causes desensitization are intervention studies. 
Kühn et al.: No Evidence for Desensitization in Empathy for Pain after a Violent Video Game

We wanted to fill this gap by conducting a longitudinal interventions study. We randomly assigned non-gamers to play one of two types of video games, either the violent video game Grand Theft Auto or the socially oriented life simulation game Sims every day for a period of two months. A passive control group was assessed with a similar battery of tests before and after this interval but received no video game intervention. Before and after as well as after another two months interval in which participants stopped playing the game, we assessed empathy for pain by means of fMRI measurements in response to photographs and videogame-like graphic depictions of the same photographs showing people hurting themselves, e.g. while cutting a cucumber. The assessment using fMRI at a later follow-up time point allows us to investigate potential long-lasting effects of violent video game exposure. According to the desensitization hypothesis decreased fMRI signal would have been anticipated in brain areas involved in processing empathy for pain [21]. Another hypothesis was that this effect may be specific for stimuli that depict pain in video-game like graphics and may not generalize to real photographs of the same scenes.

\section{Materials and Methods}

\section{Participants}

Ninety healthy participants (mean age $=28$ years, $\mathrm{SD}=7.3$, range $18-45,48$ females) were recruited by means of flyers and internet advertisements. The advertisement did mention that we were recruiting for a longitudinal study on video gaming but did not specify whether we would offer an intervention and did not mention that we were expecting training effects. Participants were randomly assigned to one of the three groups ruling out self-selection effects. The sample size was based on estimates from a previous study with a similar design [22]. After complete description of the study, the participants' informed written consent was obtained. The local ethics committee of the Charité University Clinic, Germany, approved of the study. Participants reported little, preferably no video-game usage in the past six months (none of the participants ever played the game Grand Theft Auto (GTA) or Sims in any of its versions before). The participants received financial compensation for the testing sessions (200 Euros) and performance-dependent additional payment for two behavioural tasks detailed below, but received no money for the gaming itself.

\section{Training Procedure}

The violent video game group (4 participants dropped out between pre and posttest, resulting in a group of $\mathrm{n}=26$, mean age $=27.0, \mathrm{SD}=6.1,14$ females) played the violent video game Grand Theft Auto $V$ on a Playstation 3 console over a period of eight weeks. The active control group played the non-violent video game Sims 3 on the same console (6 participants dropped out between pre and posttest, resulting in a group of $n=24$, mean age=26.1, $S D=6.9,13$ females). The passive control group (no one dropped out between pre and posttest, resulting in a group of $\mathrm{n}=30$, mean age $=30.8, \mathrm{SD}=8.3,13$ females) was not given a gaming console and had no task but underwent the same testing procedure as the two other groups. The passive control group was not aware of the fact that they were part of a control group for other participants that received active treatment, in order to prevent that they started training themselves to compensate for being randomized into the control group.

Both intervention groups were asked to train the game for approximately $30 \mathrm{~min}$ a day. However, we intentionally financially compensated the participants only for the sessions in which they came to the lab for testing. Our previous research, suggested that the perceived fun while engaging in an intervention is positively associated with outcome [22] and we therefore speculated that enforcing longer sessions and therewith forcing participants to engage longer than they felt like, might impair motivation and the potential effects of the intervention. Participants underwent a testing session before (Baseline) and after the intervention period of two month (Posttest 1) and then a follow-up testing sessions two month after the intervention period (Posttest 2). Between Posttest 1 and 2 more participants dropped out (leaving $n=22$ in the GTA group, n=23 in the Sims group, n=29 in the Control group), but we decided to base the sample on all participants that participated at Baseline and Posttest 1 to obtain maximal power in the analysis of change between Baseline and Posttest 1. 
Kühn et al.: No Evidence for Desensitization in Empathy for Pain after a Violent Video Game

Grand Theft Auto (GTA)

GTA V is an action-adventure video game situated in a fictional highly violent game world in which players are rewarded for their use of violence as a means to advance in the game. The single-player story follows three criminals and their efforts to commit heists while under pressure from a government agency. The gameplay is situated in on an open world (sandbox game) where the player can choose missions to progress an overall story, as well as engage in side activities consisting of action-adventure, driving, thirdperson shooting, occasional role-playing, stealth and racing elements. The open world design lets players freely roam in the fictitious city and countryside so that gamers could in principle decide not to commit violent acts.

The Sims 3 (Sims)

Sims is a life simulation game and also classified as a sandbox game because it lacks clearly defined goals. The player creates virtual individuals called "Sims", and customizes their appearance, their personalities and places them in a home, directs their moods, satisfies their desires and accompanies them in their daily activities and by becoming part of a social network. It offers opportunities, which the player may choose to pursue or to refuse, similar as GTA but is generally considered as a prosocial and clearly non-violent game.

\section{Scanning Procedure}

Images were collected on a Siemens Tim Trio 3T scanner (Erlangen, Germany) with a 12-channel head coil. Functional images were acquired using a T2*-weighted echo planar imaging (EPI) sequence sensitive to blood oxygen level dependent (BOLD) contrast $(\mathrm{TR}=2000 \mathrm{~ms}$, TE $=30 \mathrm{~ms}$, image matrix $=64 \times 64$,

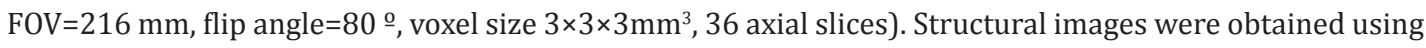
a three-dimensional T1-weighted magnetization prepared gradient-echo sequence (MPRAGE) based on the ADNI protocol (www.adni-info.org) (repetition time $=2500 \mathrm{~ms}$; echo time $=4.77 \mathrm{~ms}$; $\mathrm{TI}=1100 \mathrm{~ms}$, acquisition matrix $=256 \times 256 \times 176$, flip angle $=7^{\circ} ; 1 \times 1 \times 1 \mathrm{~mm}^{3}$ voxel size $)$.

\section{Stimuli}

We used 28 real photographs in which two hands of a female person are shown while interacting with a tool (e.g. a knife, a hammer, a stapler). In half of the pictures one hand is being hurt, in the other half this is not the case. In order to create very similar comic versions of the photographs we reworked the pictures using Adobe Photoshop in order to look similar to the graphics used in the violent video game GTA V (Fig. $1 \mathrm{~A})$.

\section{fMRI data analysis}

Functional imaging data was analyzed using Statistical Parametric Mapping software package (SPM8). EPIs were corrected for slice timing and head motion and transformed into the stereotactic normalized standard space of the Montreal Neuroimaging Institute (MNI) using the unified segmentation algorithm. Finally, EPIs were resampled (voxel size $=3 \times 3 \times 3 \mathrm{~mm}^{3}$ ) and spatially smoothed with a 3D Gaussian kernel of $8 \mathrm{~mm}$ full width at half maximum.

On the single subject level, event-related separate regressors were included for the painful photographs, the painful comic picture as well as for the not painful photographs and the not painful comic pictures. Finally, the six rigid body movement parameters were also included in the single subject GLM. Differential t-contrasts for painful versus not painful pictures across both conditions real photographs as well as videogame-like graphics at Baseline were calculated and taken to group level analysis. On the second level, these differential t-contrast images were entered into a one-sample $t$-test. Whole brain results were corrected for multiple comparisons using a family-wise error threshold of $p<0.05$ and a cluster threshold of 50 voxels. The resulting maps were overlaid onto a normalized T1 weighted MNI template (colin27) and the coordinates reported correspond to the MNI coordinate system. From the significant clusters of the whole brain contrast painful vs. not painful stimuli across both picture categories (real photographs and videogame-like graphics) of all participants at Baseline we extracted BOLD signal using MATLAB. In these brain regions that are involved in empathy for pain at Baseline we computed Condition (painful vs. not painful) $\mathrm{x}$ Group x Time and Condition x Group x Time x Stimulus category (real photographs vs. game-like graphics) interaction analyses in a repeated-measures ANOVA on the extracted values to unravel whether violent video game play influences empathy for pain. 


\section{SiGínAis}

Fig. 1. A) Examples of the stimuli used in the fMRI task. B) Significant clusters resulting from a whole brain contrast of real photographs and video-gamelike graphics pooled comparing harmful and harmless stimuli across all participants at Baseline $(\mathrm{PCC}=$ posterior cingulate cortex, $\mathrm{ACC}=$ anterior cingulate cortex, $\mathrm{dmPFC}=$ dorsal medial prefrontal cortex).
Neurosignals 2018;26:22-30

\begin{tabular}{l|l}
\hline DOI: $10.1159 / 000487217$ & (c) 2018 The Author(s). Published by S. Karger AG, Base
\end{tabular}

Published online: February 06, 2018 www.karger.com/nsg

Kühn et al.: No Evidence for Desensitization in Empathy for Pain after a Violent Video Game

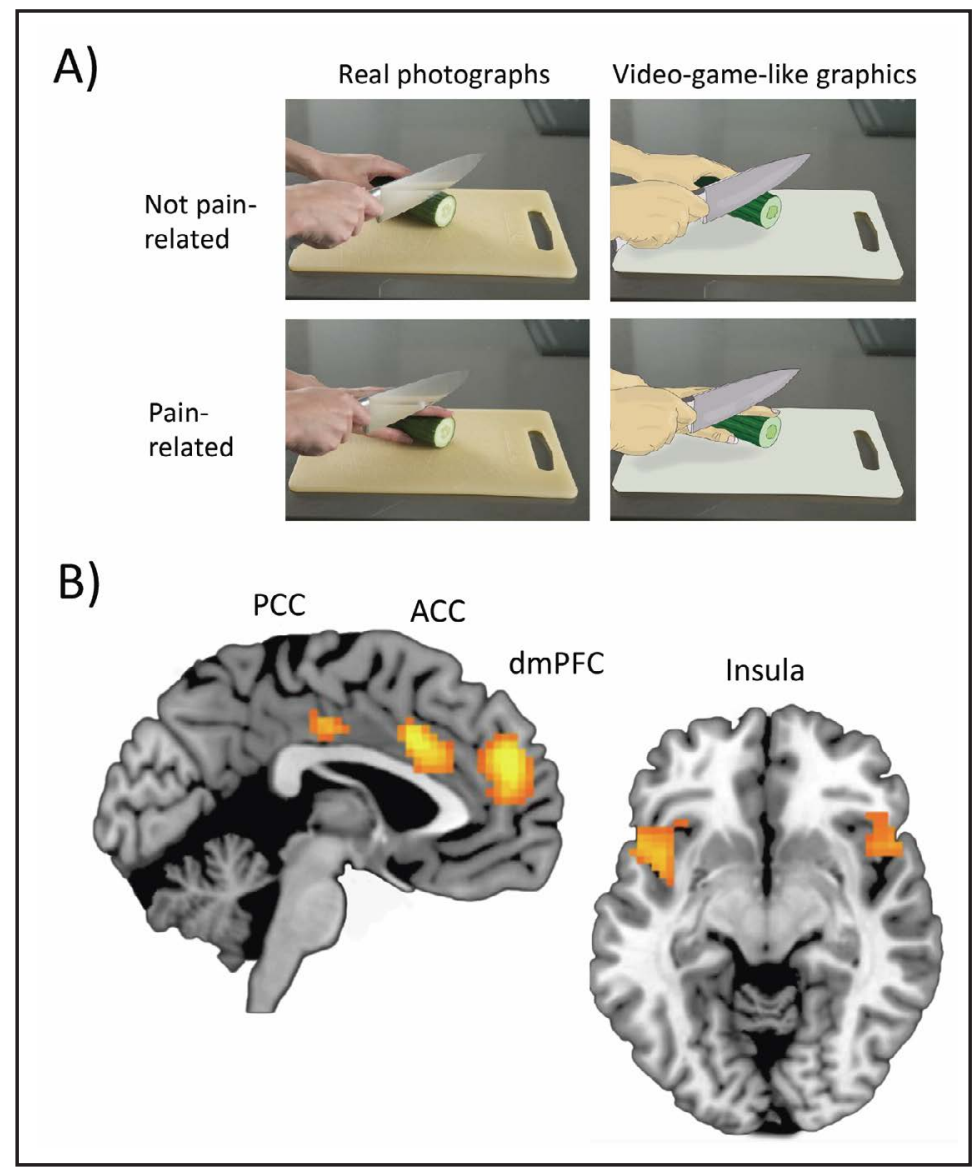

Our analysis resulted in 40 statistical tests. Setting the alpha value to 0.05 means that by pure chance two analyses should become significant. To account for this multiple testing problem and the associated alpha inflation we conducted a Bonferroni correction. According to Bonferroni the alpha value for the entire set of $\mathrm{n}$ tests is set to an alpha value of 0.05 by taking alpha/ $\mathrm{n}=0.00125$.

Since the Bonferroni correction has been criticised as overly conservative and since the two interactions of interest were not independent of one another we additionally conducted a false discovery rate (FDR) correction [23]. FDR correction also determines adjusted $p$-values for each test, however, it controls only for the number of false discoveries in those tests that result in a discovery (namely a significant result).

\section{Results}

In order to define a-priori regions of interest we computed a whole brain contrast of all painful vs. not painful stimuli (pooling across real photographs and video-game-like graphical depictions) in all participants at Baseline. In line with prior research we found a network of brain regions that have been associated with empathy for pain [21], namely bilateral anterior insula extending into inferior frontal gyrus, anterior cingulate cortex (ACC), dorsal medial prefrontal cortex (dmPFC), and posterior cingulate cortex (PCC) (Table 1, Fig. 1B).

Then we conducted interaction analyses in each of the ROIs of the empathy for pain network. Once focussing on the Condition $x$ Time $x$ Group interaction and once on the Condition x Time x Group x Stimulus type interaction. Separately for Baseline vs. Posttest 1 and Baseline vs. Posttest 2 and separately comparing GTA vs. Sims and GTA vs. Controls 
Table 1. Painful > not painful stimuli across all participants at Baseline (family-wise error corrected $p<0.05$, cluster threshold $k>50$ )

\begin{tabular}{|c|c|c|c|c|}
\hline Brain structure & $\mathrm{BA}$ & $\begin{array}{l}\text { peak coordinates } \\
\text { (MNI) }\end{array}$ & $\mathrm{T}$ (peak) & $\begin{array}{c}\text { Cluster size } \\
\text { (voxels) }\end{array}$ \\
\hline $\begin{array}{l}\text { Dorsal medial prefrontal cortex } \\
\text { (dmPFC) }\end{array}$ & $\begin{array}{l}10, \\
32\end{array}$ & $-3,50,25$ & 7.48 & 172 \\
\hline Anterior cingulate cortex (ACC) & 24 & $-3,17,34$ & 7.16 & 129 \\
\hline Posterior cingulate cortex (PCC) & 23 & $0,-16,37$ & 7.00 & 50 \\
\hline $\begin{array}{l}\text { Left anterior insula/ inferior frontal } \\
\text { gyrus }\end{array}$ & 38 & $-27,14,-20$ & 6.94 & 327 \\
\hline $\begin{array}{l}\text { Right anterior insula / inferior } \\
\text { frontal gyrus }\end{array}$ & 48 & $51,14,-2$ & 6.63 & 137 \\
\hline
\end{tabular}

Table 2. Results of the statistical ROI analysis controlling for the confounding variables age and sex. dmPFC $=$ dorsomedial prefrontal cortex, $\mathrm{ACC}=$ anterior cingulate cortex, $\mathrm{PCC}=$ posterior cingulate cortex, $\mathrm{AI}=$ anterior insula

\begin{tabular}{|c|c|c|c|c|}
\hline Dependent variables & Condition x Group x Time & $\begin{array}{l}\text { GTA vs. Sims } \\
\text { Condition } x \text { Group } x \text { Time } x \text { Stimulus type }\end{array}$ & Condition $\mathrm{x}$ Group $\mathrm{x}$ Time & $\begin{array}{l}\text { GTA vs. Controls } \\
\text { Condition } \mathrm{x} \text { Group } \mathrm{x} \text { Time } \mathrm{x} \text { Stimulus type }\end{array}$ \\
\hline $\begin{array}{l}\text { Baseline vs. Posttest } 1 \\
\text { dmPFC. }\end{array}$ & $F(1,46)=3.33, p=0.07 .5$ & $F(1,46)=0.04, p=0.84 .3$ & $F(1,52)=0.49, p=0.489$ & $F(1,52)=0.00, p=0.994$ \\
\hline $\mathrm{ACC}$ & $F(1,46)=0.41, p=0.526$ & $\mathrm{~F}(1,46)=4.08, \mathrm{p}=0.049^{*}$ & $F(1,52)=0.91, p=0.344$ & $F(1,52)=0.19, p=0.668$ \\
\hline $\mathrm{PCC}$ & $F(1,46)=1.21, p=0.278$ & $F(1,46)=0.13, p=0.726$ & $F(1,52)=0.00, p=0.984$ & $F(1,52)=0.34, p=0.565$ \\
\hline Left AI & $\mathrm{F}(1,46)=9.03, \mathrm{p}=0.004^{*}$ & $F(1,46)=0.08, p=0.781$ & $F(1,52)=1.86, p=0.179$ & $F(1,52)=0.21, p=0.646$ \\
\hline Right $\Lambda \mathrm{I}$ & $F(1,16)=0.02, p=0.881$ & $F(1,16)=0.91, p=0.336$ & $F(1,52)=1.77, p=0.189$ & $F(1,52)=0.05, p=0.821$ \\
\hline Baseline vs. Posttest 2 & & & & \\
\hline $\operatorname{dmPFC}$ & $F(1,42)=0.12, p=0.726$ & $F(1,42)=0.64, p=0.427$ & $F(1,47)=0.80, p=0.376$ & $F(1,47)=0.45, p=0.505$ \\
\hline ACC & $F(1,42)=0.01, p=0.929$ & $F(1,42)=0.00, p=0.974$ & $F(1,47)=0.00, p=0.961$ & $F(1,47)=0.82, p=0.371$ \\
\hline PCC & $F(1,42)=0.48, p=0.490$ & $F(1,42)=0.17, p=0.682$ & $F(1,47)=0.06, p=0.812$ & $F(1,47)=1.64, p=0.207$ \\
\hline Left AI & $F(1,42)=2.62, p=0.113$ & $F(1,42)=0.70, p=0.409$ & $F(1,47)=0.74, p=0.394$ & $F(1,47)=0.21, p=0.646$ \\
\hline Right AI & $F(1,42)=2.79, p=0.102$ & $F(1,42)=0.84, p=0.365$ & $F(1,47)=0.00, p=0.958$ & $F(1,47)=0.23, p=0.634$ \\
\hline
\end{tabular}

(Table 2). Neither of the results survive Bonferroni correction, nor FDR correction with a corrected threshold of $p=0.05$.

Interestingly, even the most likely randomly occurring effects with an uncorrected significance threshold do not reflect the hypothesized pattern of decreases in the empathy for pain network in the GTA training group.

The effect in left anterior insula for GTA vs. Sims in Baseline vs. Posttest 1 in the Condition $x$ Time $x$ Group interaction is driven by a change in the Sims group in which the not painful stimuli are processed with stronger insula activity at Posttest 1 compared to Baseline. This would - if anything - argue for an increase in sensitisation in the active control group rather than a decrease in the violent video game group GTA.

The effect in ACC for GTA vs. Sims in Baseline vs. Posttest 1 in the Condition x Group $x$ Time $\mathrm{x}$ Stimulus category was mainly driven by the fact that actually all Time $\mathrm{x}$ Condition interaction plots show a similar pattern, namely higher activity during pain compared to no-pain with an almost equivalent difference at Baseline vs. Posttest 1 with the exception of the real photography condition in the Sims group where the activity during pain processing decreases over time and the activity during no-pain stimuli increases over time. This pattern of the effect is likewise not in line with a desensitization in the GTA group.

In order to show that we have an adequate sensitivity to detect relevant effects we computed the effect size that we would have been able to detect. The information we used consisted of alpha error probability $=0.05$, power $=0.95$, our sample size, number groups and of measurements occasions and the correlation between the repeated measures at Posttest 1 and Posttest 2 of on average $r=0.40$. According to $G^{*}$ Power [24] our present study would enable us to detect small to medium effect sizes of 0.206 in each separate test. When accounting for the conservative Bonferroni corrected $p$-value of 0.00125 , still a small to medium effect size of 0.27 would have been detectable. 


\section{SIGENAis}

Neurosignals 2018;26:22-30

\begin{tabular}{l|l}
\hline DOI: $10.1159 / 000487217$ & C 2018 The Author(s). Published by S. Karger AG, Basel \\
Published online: February 06, 2018 & www.karger.com/nsg
\end{tabular}

Kühn et al.: No Evidence for Desensitization in Empathy for Pain after a Violent Video Game

\section{Discussion}

To our knowledge this is the first study investigating whether long-term violent video game play ( 8 weeks) causes desensitization effects in the empathy for pain network of the brain. Previous studies have either focused on the effects of playing violent games for several minutes to hours only, or compared habitual game players against controls cross-sectionally. Based on the latter database of studies a task force of the American Psychological Association recently summarized the findings on violent video games as indicating that they pose a risk factor for adverse outcomes, including increased desensitization, and decreased empathy [25]. The present findings of this study clearly contradict this conclusion. Our longitudinal interventional study design allows us to draw causal conclusions and does not show any evidence for a desensitization effect in brain signal during empathy for pain.

We randomly assigned participants, who did not report to be frequent gamers, to one of two video games that we asked them to play for $30 \mathrm{~min} /$ day for a period of two months. One group played the violent video game Grand Theft Auto $V$ and the other group played the comparably social simulation game The Sims 3. A third passive control group was recruited and tested at the same points in time but received no intervention. Before (Baseline) the two months training intervention and afterwards (Posttest 1), as well as at a follow-up appointment two months after the training intervention ended (Posttest 2) participants performed a passive viewing task in an fMRI scanner where they were confronted with painful (e.g. someone cutting herself with a knife) and non-painful stimuli either as real photographs or adapted to resemble the video-game like graphics of GTA. In order to derive the overall empathy of pain network we compared painful against non-painful stimuli at Baseline, pooling participants across all three groups. The network consisted of dmPFC, ACC, PCC and bilateral anterior insula extending into inferior frontal gyrus. This is very much in line with a previous meta-analysis on fMRI data summarizing studies of perceived pain and empathy for pain [21], where activation was most consistently observed in ACC, bilateral insula extending into inferior frontal gyrus and the dmPFC. The PCC/precuneus was also mentioned in this meta-analysis but interestingly in an analysis that focussed on study contrast where others are in pain vs. not in pain and this pain was signalled cue-based rather than based on pictures. Maybe we find PCC with our picture set because it is less explicit then the stimuli used in previous studies to depict pain. We did e.g. not show blood as a result of cutting or any bruises etc., so that participants needed to infer that the situation was painful for the observed other.

According to the desensitization account we would have expected a decrease of BOLD signal in the identified empathy of pain network over time, that is caused by the training intervention, exclusively in the group that played the violent video game. Whether these effects would be expected to occur between Baseline and Posttest 1 immediately after the training intervention, or with a delay of two months after the intervention was unclear. Moreover we speculated that the effect could be different depending on stimulus type, namely whether the stimulus was a real photography or looked more like a cartoon taken from a video game. Therefore we computed the following two interactions of interest: Condition (painful vs. not painful) x Group x Time as well as Condition (painful vs. not painful) x Group $\mathrm{x}$ Time $\mathrm{x}$ Stimulus type (real photographs vs. game-like graphics). We did this separately comparing the GTA group once against the active control group (Sims) and the passive control group. In the resulting 40 (5 ROIs $\times 2$ interactions $\times 4$ Time/Group combinations) statistical tests we did not find any significant interaction that survived multiple comparison correction. This result fits well to several previous studies referred to in the introduction that have shown no desensitization effects when comparing habitual violent video gamers against control participants using fMRI [17-20] or electroencephalography [15]. Moreover the result is in line with at least some previous findings in studies examining the effects of short-term violent video gaming $[13,14]$. However, to our opinion these studies using shortterm interventions only reflect short-lived priming effects that are observable minutes after 
game play and what research and society should be more interested in are more persistent effects of long-term violent video gaming.

\section{Conclusion}

To summarize, our study is the first longitudinal long-term intervention study investigating potential desensitization effects in empathy for pain caused by long-term violent video game play. The present evidence argues against the desensitization account proposed by the General Aggression Model [3] and clearly stands against the recent conclusions of a task force by the American Psychological Association that violent videogames increase desensitization and therefore pose a risk factor for adverse outcomes [25].

\section{Acknowledgement}

SK has been funded by a Heisenberg grant from the German Science Foundation (DFG KU 3322/1-1, SFB 936/C7), the European Union (ERC-2016-StG-Self-Control-677804) and a Fellowship from the Jacobs Foundation (JRF 2016-2018). We would like to thank Armin Junge who helped in creating the GTA-like graphics and took the pictures.

\section{Disclosure Statement}

The authors report no conflict of interest.

\section{References}

1 Anderson CA, Bushman BJ: Human aggression. Annu Rev Psychol 2002;53:27-51.

-2 Anderson CA, Bushman BJ: Effects of violent video games on aggressive behavior, aggressive cognition, aggressive affect, physiological arousal, and prosocial behavior: a meta-analytic review of the scientific literature. Psychol Sci 2001;12:353-359.

-3 Anderson CA, Berkowitz L, Donnerstein E, Huesmann LR, Johnson JD, Linz D, Malamuth NM, Wartella E: The Influence of Media Violence on Youth. Psychol Sci Public Interest 2003;4:81-110.

4 Huesmann LR, Moise-Titus J, Podolski CL, Eron LD: Longitudinal relations between children's exposure to TV violence and their aggressive and violent behavior in young adulthood: 1977-1992. Dev Psychol 2003;39:201-221.

5 Ferguson CJ, Kilburn J: Much ado about nothing: the misestimation and overinterpretation of violent video game effects in eastern and western nations: comment on Anderson et al. (2010). Psychol Bull 2010;136:174-178; discussion 182-177.

6 Ferguson CJ: Blazing Angels or Resident Evil? Can violent video games be a force for good? Rev General Psychol 2010;14:68-81.

7 Ferguson CJ: Pay No Attention to That Data Behind the Curtain: On Angry Birds, Happy Children, Scholarly Squabbles, Publication Bias, and Why Betas Rule Metas. Perspect Psychol Sci 2015;10:683691.

-8 Greitemeyer T, Mugge D0: Video games do affect social outcomes: a meta-analytic review of the effects of violent and prosocial video game play. Pers Soc Psychol Bull 2014;40:578-589.

-9 Ferguson CJ: Do Angry Birds Make for Angry Children? A Meta-Analysis of Video Game Influences on Children's and Adolescents' Aggression, Mental Health, Prosocial Behavior, and Academic Performance. Perspect Psychol Sci 2015;10:646-666. 
10 Carnagey NL, Anderson CA, Bushman BJ: The effect of video game violence on physiological desensitization to real-life violence. J Exp Soc Psychol 2007;43:489-496.

11 Staude-Müller F, Bliesener T, Luthmann S: Hostile and Hardened? An Experimental Study on (De-) Sensitization to Violence and Suffering Through Playing Video Games Swiss J Psychol 2008;67:41-50.

12 Engelhardt CR, Bartholow BD, Kerr GT, Bushman BJ: This is your brain on violent video games: neural desensitization to violence predicts increased aggression following violent video game exposure. J Exp Soc Psychol 2011;47:1033-1036.

13 Ballard M, Visser K, Jocoy K: Social context and video game play: impact on cardiovascular and affective responses. Mass Communicat Soc 2012;15:875-898.

14 Chittaro L, Sioni R: Killing non-human animals in video games: A study on user experience and desensitization to violence aspects. Psych Nology J 10:215-243.

-15 Bartholow BD, Bushman BJ, Sestir MA: Chronic violent video game exposure and desensitization to violence: behavioral and event-related brain potential data. J Exp Soc Psychol 2006;42:532-539.

-16 Montag C, Weber B, Trautner P, Newport B, Markett S, Walter NT, Felten A, Reuter M: Does excessive play of violent first-person-shooter-video-games dampen brain activity in response to emotional stimuli? Biol Psychol 2012;89:107-111.

-17 Szycik GR, Mohammadi B, Hake M, Kneer J, Samii A, Munte TF, Te Wildt BT: Excessive users of violent video games do not show emotional desensitization: an fMRI study. Brain Imaging Behav 2017;11:736743.

18 Szycik GR, Mohammadi B, Munte TF, Te Wildt BT: Lack of Evidence That Neural Empathic Responses Are Blunted in Excessive Users of Violent Video Games: An fMRI Study. Front Psychol 2017;8:174.

-19 Gao X, Pan W, Li C, Weng L, Yao M, Chen A: Long-Time Exposure to Violent Video Games Does Not Show Desensitization on Empathy for Pain: An fMRI Study. Front Psychol 2017;8:650.

-20 Regenbogen C, Herrmann M, Fehr T: The neural processing of voluntary completed, real and virtual violent and nonviolent computer game scenarios displaying predefined actions in gamers and nongamers. Soc Neurosci 2010;5:221-240.

-21 Lamm C, Decety J, Singer T: Meta-analytic evidence for common and distinct neural networks associated with directly experienced pain and empathy for pain. Neuroimage 2011;54:2492-2502.

-22 Kühn S, Gleich T, Lorenz RC, Lindenberger U, Gallinat J: Playing Super Mario induces structural brain plasticity: gray matter changes resulting from training with a commercial video game. Mol Psychiatry 2014:265-271.

23 Benjamini Y, Hochberg Y: Controlling the False Discovery Rate: A Practical and Powerful Approach to Multiple Testing J Royal Statist Soc 1995;57:289-300.

24 Faul F, Erdfelder E, Lang A-G, Buchner A: G*Power 3: A flexible statistical power analysis program for the social, behavioral, and biomedical sciences. Behavior Research Methods. Springer-Verlag, 2007, pp 175-191.

-25 Calvert SL, Appelbaum M, Dodge KA, Graham S, Nagayama Hall GC, Hamby S, Fasig-Caldwell LG, Citkowicz M, Galloway DP, Hedges LV: The American Psychological Association Task Force assessment of violent video games: Science in the service of public interest. Am Psychol 2017;72:126-143. 